\title{
LETTERS
}

doi:10.1017/S1041610212000464

\section{Insulin-like growth factor 1 and delirium}

The paper by Morandi et al. (2011), entitled "Insulin-like growth factor-1 and delirium in critically ill mechanically ventilated patients: a preliminary investigation," is of great interest due to its lack of finding a correlation between serum levels of insulin-like growth factor-1 (IFG-1) and delirium in intensive care unit (ICU) patients.

As reviewed recently by Adamis and Meagher (2011), a large body of data supports the involvement of IGF-1 in the pathogenesis of delirium. A role for IGF-1 in delirium was first suggested by Wilson et al. (2005). These investigators measured serum levels of IGF-1 in 100 acutely ill medical inpatients at the time of their admission and found that the 12 patients who later developed delirium had lower levels of IGF-1 (OR: 0.822, CI: $0.69,0.97, p=0.027)$. A role for lower levels of circulating IGF-1 in delirium has been confirmed by two studies from Adamis and Meagher (2011) and a study from our laboratory on postoperative delirium in knee replacement patients (Kwatra and Rivelli, 2008).

From the foregoing, it is clear that a lack of an association between IGF-1 and delirium needs to be scrutinized. To their credit, Morandi et al. point out several factors that could have affected their not finding an association between circulating IGF-1 levels and delirium. However, one factor that Morandi et al. did not address is the nature of the IGF-1 assay that they employed. From the work

doi:10.1017/S1041610212000786

\section{Insulin-like growth factor-1 and delirium: authors' response}

We read with great interest the comments from Motosko and colleagues (2012) regarding our paper, in which we reported that insulin-like growth factor-1 (IGF-1) was not associated with delirium in our prospective study of critically ill patients (Morandi et al., 2011). Based on these results and those of the other four published studies examining IGF-1 and delirium, we take issue with the statement that a large body of evidence supports IGF-1's role in delirium pathogenesis. As of endocrinologists, we know that measuring IGF1 in plasma/serum is full of pitfalls, and proper validation of the assay is needed. Unfortunately, Morandi et al. do not provide any details of the IGF-1 assay they used, and it is important to know whether they validated the assay according to the guidelines provided by Frystyk et al. (2010).

\section{References}

Adamis, D. and Meagher, D. (2011). Insulin-like growth factor I and the pathogenesis of delirium: a review of current evidence. Fournal of Aging Research, doi: $10.4061 / 2011 / 951403$.

Frystyk, J., Freda, P. and Clemmons, D. R. (2010). The current status of IGF-I assays - a 2009 update. Growth Hormone IGF Research, 20, 8-18.

Kwatra, M. and Rivelli, S. (2008). Baseline Plasma Igf-1 Levels Relate to Postoperative Delirium in Knee Arthroplasty Patients. Orlando, FL: American Society of Anesthesiologists.

Morandi, A. et al. (2011). Insulin-like growth factor-1 and delirium in critically ill mechanically ventilated patients: a preliminary investigation. International Psychogeriatrics, 23, 1175-1181.

Wilson, K. et al. (2005). Plasma insulin growth factor-1 and incident delirium in older people. International fournal of Geriatric Psychiatry, 20, 154-159.

CATHERINe MOTOSKo, KRISTINE BRown AND MADAN KWATRA

Department of Anesthesiology, Duke University, Durham, North Carolina, USA

Email: madan.kwatra@duke.edu pointed out by Adamis and Meagher (2011) in their recent systematic review, these five studies were small and produced conflicting results, with only three of the five finding an association between IGF-1 and delirium. Important differences in the patients studied may explain, in part, the conflicting results. Investigations of older medical inpatients who were not critically ill found IGF-1 to be associated with delirium, but neither a study of hip surgery patients (Lemstra et al., 2008) nor our study of intensive care unit (ICU) patients found an association. The relative importance of IGF-1 to delirium might be diminished during critical illness given that a multitude of risk factors are involved in the pathogenesis of delirium in this vulnerable 
population. On average, medical ICU patients are subject to more than ten delirium risk factors; changes in IGF-1 in this setting might therefore contribute less to delirium than in other settings.

Appropriate validation and use of the assay employed to analyze levels of IGF-1, as highlighted by Motosko and colleagues, is essential when studying this hormone. Similar to the four studies that preceded ours, our study used a commercially available IGF-1 assay (ALPCO Diagnostics; Salem, NH, USA). The characteristics and validation of this radioimmunoassay which is calibrated against the WHO International Reference Standard preparation of IGF1 (NIBSC Code 02/254) and was designed to avoid errors caused by IGF binding protein interference - are described on the ALPCO Diagnostics website (http://www.alpco.com/pdfs/22/22IGF-R21.pdf). The Hormone Assay and Analytical Services Core at Vanderbilt University, where our samples were assayed, has extensive experience with the ALPCO kit; before using the assay, the Core lab established inter- and intra-assay coefficients of variability, confirmed that varying concentrations of exogenous IGF-1 could be accurately recovered, and ran a set of controls. Though we agree that the additional procedures recommended by Frystyk et al. (Frystyk et al., 2010) are reasonable suggestions, a consensus guideline for IGF-1 assays has yet to be established and none of the published studies of IGF-1 and delirium reported adherence to these suggestions.

Our finding that IGF-1 concentrations were not associated with delirium during critical illness does not rule out the possibility that IGF-1 plays a role in the development of cognitive deficits affecting ICU patients. Long-term cognitive impairment, for example, is common among ICU survivors (Hopkins and Jackson, 2006) and may be associated with derangements in IGF-1. Motosko and colleagues' comments are important and should lead to more thorough reporting in future publications regarding the role of IGF- 1 in neurologic outcomes of critically ill patients.

\section{Conflict of interest}

None.

\section{Description of authors' roles}

Morandi drafted and critically revised the manuscript. He also obtained the final approval of manuscript from all the authors.

\section{References}

Adamis, D. and Meagher, D. (2011). Insulin-like growth factor I and the pathogenesis of delirium: a review of current evidence. Fournal of Aging Research, online journal; doi: $10.4061 / 2011 / 951403$.

Frystyk, J., Freda, P. and Clemmons, D. R. (2010). The current status of IGF-1 assays: a 2009 update. Growth Hormone and IGF Research, 20, 8-18.

Hopkins, R. O. and Jackson, J. C. (2006). Long-term neurocognitive function after critical illness. Chest, 130, 869-878.

Lemstra, A. W., Kalisvaart, K. J., Vreeswijk, R., van Gool, W. A. and Eikelenboom, P. (2008). Pre-operative inflammatory markers and the risk of postoperative delirium in elderly patients. International fournal of Geriatric Psychiatry, 23, 943-948.

Morandi, A. et al. (2011). Insulin-like growth factor-1 and delirium in critically ill mechanically ventilated patients: a preliminary investigation. International Psychogeriatrics, 23, 1175-1181.

Motosko, C., Brown, K. and Kwatra, M. (2012). Insulin-like growth factor 1 and delirium (Letter). International Psychogeriatrics, epublished ahead of print, doi: $10.1017 /$ S1041610212000464.

\section{A. Morandi ${ }^{1,2,3}$ AND T. D. GIRARD ${ }^{3,4,5,6}$}

${ }^{1}$ Rehabilitation and Aged Care Unit Hospital, Ancelle, Cremona, Italy

${ }^{2}$ Geriatric Research Group, Brescia, Italy

${ }^{3}$ Center for Quality Aging, Nashville, Tennessee, USA

${ }^{4}$ Center for Health Services Research, Nashville, Tennesse, USA

${ }^{5}$ Division of Allergy, Pulmonary, and Critical Care Medicine in the Department of Medicine at the Vanderbilt University School of Medicine, Nashville, Tennessee, USA

${ }^{6}$ Geriatric Research, Education and Clinical Center (GRECC) Service, Department of Veterans Affairs Medical Center, Tennessee Valley Healthcare System, Nashville, Tennessee, USA Email: morandi.alessandro@gmail.com 